\title{
Índice de Calidad Ecológica y gestión hídrica en tres ecosistemas de Colombia
}

\author{
Esnedy Hernández ${ }^{* 1}$, Diana María Agudeloํㅡㄹ Elizabeth Flórez ${ }^{1}$, Robinson Sepúlveda ${ }^{1}$, \\ Ana María Zabala ${ }^{1}$, Lady Vargas ${ }^{2}$, Mateo Parra ${ }^{1}$, Giovanny Ramírez ${ }^{2}$, Néstor Aguirre ${ }^{1}$ \\ \& Fabio Vélez ${ }^{1}$ \\ 1. Grupo GeoLimna, Facultad de Ingeniería, Universidad de Antioquia, Medellín, Colombia; \\ esnedy.hernandez@udea.edu.co,diana.agudeloe@udea.edu.co,elizabeth.florez1@udea.edu.co, \\ robinsepul.rs@gmail.com, ana.zabala@udea.edu.co,mateo.parra@udea.edu.co,nestor.aguirre@udea.edu.co, \\ fabio.velez@udea.edu.co \\ 2. Instituto Investigaciones Ambientales del Pacífico, Chocó, Colombia; lvargas@iiiap.org.co, gramirez@iiap.org.co \\ * Correspondencia
}

Recibido 22-IV-2020. Corregido 20-VIII-2020. Aceptado 14-IX-2020.

\begin{abstract}
Ecological quality index for water management in three Colombian ecosystems. Introduction: Research and diagnosis of water sources is of academic and governmental interest, for this reason, the exploration of numerical tools applied to watershed management, offers the possibility to identify where and what variables are useful in monitoring and rehabilitation programs of aquatic ecosystems. Environmental planning and management, which includes water quality, is conventionally analyzed by physical, chemical and hydrobiological indexes. In 2014, through the water management guide, included the assessment of the ecological quality index (EQI); it was generated a comprehensive approach through a numerical system of correlations that diagnoses, classifies and detects environmental impacts. Objective: This research aims to demonstrate that the EQI allows to assess the quality of aquatic ecosystems affected by different environmental situations. Methods: In order to analyze the application of this tool, we studied three scenarios located in different biogeographical regions of Colombia and, as a hydrobiological group indicator, we used peripheral and phytoplankton algae. The ecosystems were monitored between 2007 and 2015 and correspond to a flood plain, a high mountain Andean river and a group of water stream in a tropical humid forest. Results: Canonical correlations were significant $(\mathrm{P}<0.005)$ and a model of weighted averages, allowed to estimate the optimum and the tolerance of each taxa for the sites ecological classification; variables related to ion concentration, acidity damping, temperature and hydraulics, influenced the models that explained the abundances distribution of the studied biological groups. Conclusions: The application of the EQI makes it possible to identify variables, organisms and numerical systems to classify ecological status. These results are useful in the diagnosis and monitoring of aquatic ecosystems and that can be implemented in other scenarios.
\end{abstract}

Key words: ecological quality; periphytic algae; phytoplanktonic algae; ecological indicators.

Hernández, E., Agudelo, D.M., Flórez, E., Sepúlveda, R., Zabala, A.M., Vargas, L., Parra, M., Ramírez, G., Aguirre, N., \& Vélez, F. (2020). Índice de Calidad Ecológica y gestión hídrica en tres ecosistemas de Colombia. Revista de Biología Tropical, 68(4), 1337-1345.

El enfoque de la gestión integral del recurso hídrico promueve el desarrollo y gestión coordinada del agua, la tierra y las demandas de las poblaciones con el propósito de maximizar el bienestar económico y social sin comprometer la sostenibilidad de ecosistemas vitales (MADS, 2018). El deterioro de los ecosistemas ligado al crecimiento demográfico, el desarrollo económico y el cambio climático (Global Water Partnership, 2009). 
han conducido a medidas relacionadas con la planificación y gestión ambiental en Colombia (MADVT, 2010), como las guías para el Plan de Ordenación y Manejo de Cuencas Hidrográficas (POMCA) y el Plan de Ordenamiento del Recurso Hídrico (PORH), instrumentos que incorporan lineamientos para evaluar la demanda, calidad y riesgo del recurso hídrico.

En el 2014, la guía para la ejecución de los PORH retomó aportes e insumos técnicos, e incluyó la aplicación de nuevos índices de calidad física y química e hidrobiológica (MADS, 2014; MADS, 2018), con el fin de unificar los análisis de calidad del agua. Para lograr este propósito se postuló el uso de un índice publicado por Haase \& Nolte (2008), quienes desarrollaron un indicador numérico que define la variación biológica de una comunidad en función del componente ambiental (Chalar, Arocena, Pacheco, \& Fabián, 2011).

El Índice de Calidad Ecológica (ICE) permite definir un gradiente ambiental de acuerdo con las particularidades físicas y químicas que presenta cada sistema y, a su vez, determinar valores óptimos y de tolerancia de cada organismo, sin condicionar el grupo y el sistema al que pertenecen, en función de dicho gradiente (Flórez-Córdoba, 2019; Braak \& Šmilauer, 2002; Forero, Longo, Ramírez, Jairo, $\&$ Chalar, 2014).

El primer ejercicio en investigación que usó oficialmente esta metodología en Colombia, fue realizado por Forero et al. (2014) en la cuenca del Río Negro-Antioquia con macroinvertebrados. Posteriormente Hernández et al. (2018) lo aplicaron en el Río San Juan-Antioquia, en este caso, usando algas perifíticas.

Este trabajo recopila la información secundaria de diferentes investigaciones. Se analizó un río Andino de alta montaña, una planicie de inundación del Caribe y un grupo de quebradas en el Chocó biogeográfico. La evaluación contempla sitios perturbados por agricultura, minería y aguas residuales y pretende demostrar que el índice es efectivo para evaluar diferentes estresores ambientales. El análisis ecológico detectó las variables y organismos indicadores en cada sistema por medio de un ejercicio de clasificación que permite identificar grupos y posterior seguimiento del estado ecológico en estos ambientes.

\section{MATERIALES Y MÉTODOS}

Sistemas de estudio: Se analizaron tres sistemas acuáticos ubicados en los departamentos de Antioquia, Chocó y Córdoba, Colombia. En primer lugar, se encuentra la cuenca del río San Juan, ubicada en el suroeste del departamento de Antioquia en la región Andina, con una extensión aproximada de $1819 \mathrm{~km}^{2}$ y su corriente principal es el río San Juan que va desde los 1851 a 571 m.s.n.m., pasando por tierras que presentan agricultura extensiva por monocultivos de café. El segundo lugar se encuentra en la región Pacífica, Distrito Minero del municipio de Condoto, Chocó, específicamente en el corregimiento Jigualito, que se encuentra entre los 66 y 99 m.s.n.m., donde se analizó un grupo de quebradas afectadas por diferentes intervalos de minería desde hace más de 30 años. Por último, se consideró la planicie de inundación de Ayapel ubicada en el extremo oriental del Departamento de Córdoba en la región Caribe, sistema que se encuentra altamente impactado por la minería, agricultura y ganadería. En cada uno de estos ecosistemas fueron ubicados entre seis (Río San Juan) a ocho (Quebradas Jigualito y planicie inundación Ayapel) estaciones de muestreo, los cuales incluyen sitios libres del agente estresante y sitios altamente afectados. Los muestreos fueron realizados entre el 2007 y 2016.

Variables ambientales: Por medio de un equipo multiparamétrico $\mathrm{HACH} \mathrm{HQ} 40 \mathrm{~d}$ provisto de sensores in situ, se determinaron temperatura ambiente y del agua $\left({ }^{\circ} \mathrm{C}\right)$, oxígeno disuelto $(\mathrm{mg} / \mathrm{L}), \mathrm{pH}$, conductividad eléctrica $(\mu \mathrm{S} / \mathrm{cm})$ y sólidos disueltos $(\mathrm{mg} / \mathrm{L})$. La cuantificación de la turbidez (NTU), alcalinidad y dureza total $\left(\mathrm{mg} / \mathrm{L} \mathrm{CaCO}_{3}\right)$ y concentraciones de nutrientes $(\mathrm{mg} / \mathrm{L})$ : nitrógeno total, nitritos, nitratos, nitrógeno amoniacal, fósforo total y ortofosfatos, las cuales se realizaron por medio de un fotómetro modelo compacto PF-12. 
Grupos hidrobiológicos: En este trabajo se consideraron dos grupos hidrobiológicos: algas perifíticas en el Río San Juan y las quebradas de Jigualito (Antioquia y Chocó respectivamente) y algas fitoplanctónicas en la planicie de inundación de Ayapel (Caribe), el análisis incluyo un número de muestras de 24, 32 y 34 para cada sistema, respectivamente. La toma de algas perifíticas en los ríos de Antioquia y Chocó se realizó por raspado del material adherido a sustratos (piedras, troncos, hojarasca) inmersos en el lecho de la corriente usando un cuadrante de $8 \mathrm{~cm}^{2}, 30$ veces en cada estación. Las muestras de fitoplancton se tomaron en por medio de una muestra integrada de la zona fótica a través de una botella tipo Kemmerer. Las muestras de agua en cada caso fueron empacadas en recipientes plásticos y fijadas con $5 \mathrm{ml}$ de solución de Lugol al $10 \%$.

Análisis de laboratorio: Las muestras se observaron a través de un microscopio invertido provisto de una reglilla ocular y una cámara de conteo. Las observaciones se realizan con un aumento de $400 \mathrm{X}$, para la determinación de la densidad por mililitro se aplicó la expresión de Ros (Ros, 1979). Las determinaciones se realizaron con las claves taxonómicas de Ettl, Gerloff, \& Heynig (1977); Huber-Pestalozzi (1961); Komárek, Anagnostidis, Pascher, Ettl, \& Büdel (1999); Prescott, Croasdale, Vinyard, \& Bicudo (1981); Ramírez (2000) y asistencia de especialistas.

Análisis de los datos: En primer lugar, se realiza un análisis descriptivo de las variables. Posteriormente, para el cálculo del ICE, propuesto por Haase \& Nolte (2008) y adaptado por Chalar et al. (2011), se consideran cuatro pasos fundamentales: 1) Determinación del gradiente ambiental (Braak \& Šmilauer, 2002), 2) estandarización de los puntajes de las estaciones en el primer eje canónico del modelo, 3) cálculo de los valores óptimos y de tolerancia para cada taxón, 4) cálculo del ICE por estación $=\left(\Sigma\right.$ Opi $^{*}$ Toli $*$ Abi $) /\left(\left(\Sigma\right.\right.$ Opi ${ }^{*}$ Toli*Abi $)$; donde (Opi) es el valor óptimo, (Toli) el valor de tolerancia y (Abi) la abundancia, en este paso se incluye el agrupamiento de las estaciones (método de Ward y distancia euclidiana); por último se presentan los mayores coeficientes de correlación de Spearman entre el índice con las variables físicas y químicas (Tabla 1). Para el procesamiento y análisis de la información, se utilizaron los resultados de los parámetros físicos, químicos, hidráulicos y biológicos considerados en las investigaciones de Hernández et al. (2018), Vargas-Porras (2016) y Zabala-Agudelo (2016).

\section{RESULTADOS}

Algas perifíticas, fitoplanctónicas y variables ambientales: Un número de $35 \mathrm{y}$ 52 taxa de algas perifíticas fueron registradas en los ríos San Juan y Jigualito, ubicados en Antioquia y Chocó respectivamente. Por su parte, en el sistema cenagoso de Ayapel (Córdoba) se cuantificaron 44 morfotipos de algas fitoplanctónicas. En los tres casos, la mayoría de los taxa pertenecen a las divisiones Cianobacterias, Clorofíceas, Euglenofíceas y la división con mayor número de representantes corresponde a la división Bacillariofíceas o diatomeas. Entre los ecosistemas, la escala de densidades se encuentra entre 22 y 3392 (ind $/ \mathrm{cm}^{2}$ ) y de riqueza de taxa entre 10 y 17 . Con respecto a las variables ambientales, los sistemas difieren principalmente en la concentración de iones o presencia de material disuelto o suspendido, situación reflejada por variables como la conductividad (13-75 $\mu \mathrm{S} / \mathrm{cm}$ ) y la turbidez (39-119 UNT), parámetros que fueron más elevados en la ciénaga de Ayapel en contraste con los Ríos San Juan y Jigualito. Otras variables como la concentración de oxígeno disuelto (5.2-7.7 $\mathrm{mg} / \mathrm{L})$, el $\mathrm{pH}(6.3-7.3)$ y la concentración de nitratos, amonio y ortofosfatos no presentaron diferencias importantes entre los sistemas y sus valores indican ambientes de condiciones oligo a mesotróficas (Tabla 1).

Determinación del gradiente ambiental: La longitud del gradiente del primer eje es superior a 2.5 en los tres casos, por lo cual se indica que el método de ordenación 
TABLA 1

Media y coeficiente de variación de las variables ambientales y las densidades y riquezas de las comunidades hidrobiológicas

TABLE 1

Mean and coefficient of variation of the environmental variables and the densities and richness of the hydrobiological communities

\begin{tabular}{|c|c|c|c|}
\hline \multirow[b]{2}{*}{ Variables } & \multicolumn{3}{|c|}{ Sistema analizado y referencia bibliográfica } \\
\hline & $\begin{array}{l}\text { Río San Juan } \\
\text { (Hernández et al., 2018) }\end{array}$ & $\begin{array}{c}\text { Ciénaga de Ayapel } \\
\text { (Zabala-Agudelo, 2016) }\end{array}$ & $\begin{array}{l}\text { Grupo de las Quebradas Jigualito } \\
\text { (Vargas-Porras, 2016) }\end{array}$ \\
\hline Velocidad (m/s) & & & $0.37(92.57 \%)$ \\
\hline Caudal $\left(\mathrm{m}^{3} / \mathrm{s}\right)$ & & & $0.41(98.25 \%)$ \\
\hline Temperatura ambiente $\left({ }^{\circ} \mathrm{C}\right)$ & $25.92(13.26 \%)$ & & \\
\hline Temperatura del agua $\left({ }^{\circ} \mathrm{C}\right)$ & $20.94(9.02 \%)$ & $31.2(3.02 \%)$ & $27.56(6.04 \%)$ \\
\hline Oxígeno disuelto (mg/L) & $7.71(6.11 \%)$ & $5.53(26.07 \%)$ & $5.25(22.34 \%)$ \\
\hline Saturación oxígeno disuelto (\%) & $99.73(4.10 \%)$ & & \\
\hline Conductividad eléctrica $(\mu \mathrm{S} / \mathrm{cm})$ & $75.40(24.33 \%)$ & $66.28(55.64 \%)$ & $13.25(87.50 \%)$ \\
\hline $\mathrm{pH}$ del agua $(\mathrm{U}$ de $\mathrm{pH})$ & $7.26(6.74 \%)$ & $7.07(7.61 \%)$ & $6.31(8.92 \%)$ \\
\hline Turbiedad (UNT) & $38.72(90.86 \%)$ & $118.78(23.17 \%)$ & $69.78(284.38 \%)$ \\
\hline Alcalinidad (mg/L) & $0.68(32.18 \%)$ & & \\
\hline Nitritos - $\mathrm{NO}_{2}(\mathrm{mg} / \mathrm{L})$ & & & $0.03(187.00 \%)$ \\
\hline Dureza $(\mathrm{mg} / \mathrm{L})$ & $42.75(35.53 \%)$ & $23.61(45.04 \%)$ & \\
\hline Nitratos - $\mathrm{NO}_{3}(\mathrm{mg} / \mathrm{L})$ & $2.07(70.66 \%)$ & $2.14(125.56 \%)$ & $2.84(210.80 \%)$ \\
\hline Nitrógeno amoniacal - NH4 (mg/L) & $0.12(64.10 \%)$ & $0.20(113.94 \%)$ & \\
\hline Fosfatos $-\mathrm{PO}_{4}(\mathrm{mg} / \mathrm{L})$ & $0.43(27.97 \%)$ & $0.47(105.97 \%)$ & $0.54(123.43 \%)$ \\
\hline Nitrógeno total (mg/L) & $0.60(35.78 \%)$ & & \\
\hline Fósforo total (mg/L) & $0.19(70.49 \%)$ & & \\
\hline Demanda química de oxígeno (mg/L) & $14.00(49.76 \%)$ & & \\
\hline Potencial REDOX (mV) & & $208.47(20.99 \%)$ & \\
\hline Sólidos disueltos totales (mg/L) & & & $6.85(81.13 \%)$ \\
\hline Sólidos suspendidos $(\mathrm{mg} / \mathrm{L})$ & & & $74.47(258.77 \%)$ \\
\hline $\begin{array}{l}\text { Algas perifíticas }\left(\mathrm{org} / \mathrm{cm}^{2}\right) \text { y } \\
\text { fitoplanctónicas }(\mathrm{org} / \mathrm{ml})\end{array}$ & $3392(132.5 \%)$ & $22(148.58 \%)$ & $357(77.98 \%)$ \\
\hline Riqueza algas perifiticas/fitoplanctónicas & $10(41.83 \%)$ & $17(48.14 \%)$ & $11(37.69 \%)$ \\
\hline
\end{tabular}

adecuado para modelar la respuesta de los taxa al gradiente ambiental es un análisis de correspondencia canónico (ACC). El ACC fue significativo en los tres casos (valor $\mathrm{P}<0.05$ ), en todos los casos se consideró la explicación de los dos primeros ejes. En el caso del río San Juan el modelo explicó un $40.4 \%$ del total de la varianza y las variables que más influenciaron la distribución de las algas perifíticas y los sitios son la temperatura, el $\mathrm{pH}$, los sólidos disueltos y los ortofosfatos (Apéndice digital 1A). En el caso del grupo de las quebradas de Jigualito en el Chocó el modelo explica el $31 \%$ y el caudal, pH, oxígeno, temperatura, concentración de nitritos y sólidos suspendidos establecieron una mayor importancia en éste (Apéndice digital 1B). En contraste, la distribución del fitoplancton en los sitios analizados en la ciénaga de Ayapel obtiene una explicación del $52.7 \%$, con la influencia de los sólidos disueltos, la dureza total, la conductividad y la concentración de nitratos y ortofosfatos (Apéndice digital 1C).

Valores óptimos y de tolerancia de los taxa: En el Apéndice digital 2 se encuentra el perfil de los óptimos y tolerancias de los taxa para cada sistema. Estos perfiles corresponden 
al puntaje que obtiene cada taxa por promedios ponderados de la abundancia en función del componente ambiental en el primer eje del ACC.

En relación a la distribución de estos perfiles en el caso del Río San Juan, El Río Jiguialito y la Ciénaga de Ayapel 27, 42 y 38 taxa respectivamente registran valores óptimos superiores a seis (Apéndice digital 2A; Apéndice digital 2B; Apéndice digital 2C), sobre estos grupos de taxa se resaltan las siguientes particularidades, en el sistema del Río San Juan nueve de los 27 taxa con altos óptimos indican baja la tolerancia a la función del factor ambiental, es el caso de los taxa: Agilaria sp., Fragilaria ulna sp., Cymbella sp., Pseudoanabanabaena sp., Terspsinoe sp., Borzia sp., Zygnema sp., Chaetophora sp., y Gomphonema sp., de ellos, los últimos 5 presentan óptimos superiores a 9 y reducen al máximo su amplitud con la tolerancia, por lo tanto son los organismos más sensibles al factor ambiental que caracteriza este sistema. Al analizar ésta misma tendencia en las Quebradas Jigualito se destacan los taxa: Oedoclonium sp., Eunotia cf. gracilis, Pinnularia viridis, Actinotanium sp., Fragillariforma sp., entre ellos, los tres últimos son los indicadores con mayor óptimo y menor tolerancia, en este sistema la mayoría de los organismos registran amplias tolerancias con respecto a su óptimo.

En contraste, en la ciénaga de Ayapel la mayoría de los taxa registran reducidas tolerancias en relación a su óptimo, los taxa Peridinium cf. volzii, Trachelomonas volcina, Oscillatoria limnetica, Peridinium cf. inconspicum, Pinnularia episcopalis, Monorraphidium contortum, Nitzschia acicularis y Closterium setaceum acentúan una reducida tolerancia, entre ellos los casos más extremos fueron: Monorraphidium contortum, Nitzschia acicularis y Closterium setaceum, estos organismos con valores óptimos superiores a nueve indican mínima tolerancia al gradiente ambiental en la Ciénaga de Ayapel.

Índice de calidad ecológico: Después de aplicar la ecuación que define el índice, se establecen tres grupos o categorías de valores (Tabla 2), las cuales son seleccionadas según las indicaciones sugeridas por Waas et al. (2014) y Chalar et al. (2011). Éstas variables (alcalinidad, ortofosfatos, sólidos disueltos, nitratos, nitritos, caudal, temperatura, oxígeno disuelto, dureza total y conductividad) generan un peso importante en los modelos del ACC de cada sistema y los valores son clasificados por análisis clúster. Este agrupamiento podría denominar tres categorías: bajos, intermedios y altos, según el valor del ICE en cada sistema En la Tabla 2, se muestra el coeficiente de correlación de Spearman y la ecuación de la regresión lineal entre las variables y el ICE.

Los tres sistemas registran relaciones inversamente proporcionales con el índice, tal fue el caso de los sólidos disueltos en el Río San Juan, la temperatura y la concentración de nitritos en el Grupo de las Quebradas Jigualito y la dureza, la concentración de nitratos, ortofosfatos y la conductividad en la ciénaga de Ayapel, estos resultados indican que ante las disminuciones del índice estas variables tienden a incrementar, en contraste, parámetros como la alcalinidad, el caudal y el oxígeno, sugieren relaciones directamente proporcionales al comportamiento del índice.

\section{DISCUSIÓN}

El análisis de la relación entre las condiciones ambientales con la distribución de los organismos a través del ICE, permitió identificar en cada uno de los tres ecosistemas, las variables que inciden en la tolerancia o amplitud ecológica a lo largo del gradiente ambiental, es decir, cómo se comporta la abundancia de los taxa ante la variación de condiciones ambientales. Variables asociadas a la concentración de iones, la amortiguación de la acidez, la temperatura y el caudal se destacan en la explicación de los modelos en los tres sistemas analizados y a través de esos gradientes de correlación, el índice potencia el análisis de dos aspectos fundamentales, las tendencias de comportamiento de los organismos en cuanto a los valores óptimos y de tolerancia y las clasificaciones 
TABLA 2

Clasificación del índice de calidad ecológica con las variables ambientales de mayor peso en los modelos del ACC por sistema, grupos con su respectivo coeficiente de correlación y modelo lineal

TABLE 2

Classification of the ecological quality index with the environmental variables of greatest weight in the CCA models by system, groups with their respective correlation coefficient and linear model

\begin{tabular}{|c|c|c|c|c|c|c|}
\hline \multirow{3}{*}{$\begin{array}{l}\text { Sistema } \\
\text { Río } \\
\text { San Juan }\end{array}$} & \multicolumn{2}{|c|}{ ICE } & \multicolumn{4}{|c|}{ Variable } \\
\hline & \multicolumn{2}{|c|}{ Grupo } & \multicolumn{2}{|c|}{ Alcalinidad (mg/L) } & \multicolumn{2}{|c|}{ Sólidos disueltos (mg/L) } \\
\hline & Bajo & $<5.8$ & \multicolumn{2}{|c|}{$<0.5$} & \multicolumn{2}{|c|}{$>102.3$} \\
\hline & Intermedio & $5.8-8.1$ & \multicolumn{2}{|c|}{$0.5-0.7$} & \multicolumn{2}{|c|}{$98.4-102.3$} \\
\hline & Alto & $>8.1$ & \multicolumn{2}{|c|}{$>0.7$} & \multicolumn{2}{|c|}{$<98$} \\
\hline & \multicolumn{2}{|c|}{ coef. S y ecuación } & \multicolumn{2}{|c|}{$(0.3) y=0.1747 x+0.7191$} & \multicolumn{2}{|c|}{$(-0.3) y=-0.074 x+0.7985$} \\
\hline \multirow{6}{*}{$\begin{array}{l}\text { Grupo de } \\
\text { las } \\
\text { Quebradas } \\
\text { Jigualito }\end{array}$} & \multicolumn{2}{|c|}{ Grupo } & Temp $\left({ }^{\circ} \mathrm{C}\right)$ & Caudal $\left(\mathrm{m}^{3} / \mathrm{s}\right)$ & $\mathrm{OD}(\mathrm{mg} / \mathrm{L})$ & $\mathrm{NO}_{2}(\mathrm{mg} / \mathrm{L})$ \\
\hline & Bajo & $<4.9$ & $>31$ & $<12.3$ & $<3.8$ & $>0.37$ \\
\hline & Intermedio & $4.9-7.1$ & $27-31$ & $12.3-50.8$ & $3.8-6.7$ & $0.05-0.37$ \\
\hline & Alto & $>7.1$ & $<27$ & $>50.8$ & $>6.7$ & $<0.05$ \\
\hline & \multirow{2}{*}{\multicolumn{2}{|c|}{ coef. S y ecuación }} & $(-0.5)^{*}$ & $(0.4)^{*}$ & $(0.4)^{*}$ & $(-0.4)^{*}$ \\
\hline & & & $y=-0.9074 x+33.963$ & $y=0.1636 x-0.7491$ & $y=0.5938 x+1.0544$ & $y=-0.0015 x+0.0448$ \\
\hline \multirow{6}{*}{$\begin{array}{l}\text { Ciénaga } \\
\text { Ayapel }\end{array}$} & \multicolumn{2}{|c|}{ Grupo } & Dureza Total (mg/L) & $\mathrm{NO}_{3}(\mathrm{mg} / \mathrm{L})$ & $\mathrm{PO}_{4}(\mathrm{mg} / \mathrm{L})$ & Conductividad $(\mu \mathrm{S} / \mathrm{cm})$ \\
\hline & Bajo & $<2.59$ & $>56.7$ & $>10.0$ & $>1.0$ & $>143.5$ \\
\hline & Intermedio & $2.59-6.72$ & $13-56.7$ & $2.8-10.0$ & $0.53-1.0$ & $38.3-143.5$ \\
\hline & Alto & $>6.72$ & $<13$ & $<2.8$ & $<0.53$ & $<38.3$ \\
\hline & \multirow{2}{*}{\multicolumn{2}{|c|}{ coef. S y ecuación }} & $(-0.6)^{*}$ & $(-0.5)^{*}$ & $(-0.4)^{*}$ & $(-0.4)^{*}$ \\
\hline & & & $y=-0.1306 x+11.827$ & $y=-0.7234 x+10.289$ & $y=-1.4083 x+9.4108$ & $y=-0.0248 x+10.389$ \\
\hline
\end{tabular}

*Valores p del coeficiente de Spearman $(\mathrm{S})<(0.05)$.

numéricas (Forero et al., 2014; Hernández et al., 2018), las cuales separan tres grupos de datos por medio de valores altos, medianos y bajos. Estas clasificaciones son la referencia cuantitativa para establecer una escala en los parámetros ambientales (Chalar et al., 2011) y ambos aspectos permiten identificar sitios con menor calidad ecológica y variables que se correlacionan con esa situación particular en cada sistema.

En el ACC que soporta el índice, las nuevas variables o componentes representan una combinación de las variables originales y un número menor de variables que explican la mayor parte de la variación (Braak \& Prentice, 1988). Entre las utilidades que brindan estos aspectos se encuentra la detección de variables de monitoreo que juegan un papel importante en la variación, y son sensibles en cada sistema, y la guía de alerta que proporciona el rango numérico de la variable (Esteves, 1998;
Riss, Ospina, \& Gutiérrez, 2002; Barakat, El Baghdadi, Rais, Aghezzaf, \& Slassi, 2016), por ejemplo; en la ciénaga de Ayapel se detectó que la concentración de iones representada por los nitratos, los ortofosfatos, la conductividad y la dureza en el agua, genera un peso importante en la clasificación de los valores del índice; por ejemplo, la conductividad por debajo de 38.3 $(\mu \mathrm{S} / \mathrm{cm})$ se asocia con altos valores del índice de calidad, aspecto relevante para éste sistema donde ese informa actividad agrícola y minera (Zabala-Agudelo, 2016).

Los conjuntos de valores bajos, intermedios y altos del índice coinciden con el comportamiento de variables asociadas a la situación que afecta el sistema, es el caso de las descargas de aguas residuales y cultivos de café en el caso del Río San Juan-Antioquia, en el cual los sólidos disueltos y la alcalinidad demuestran relaciones importantes con el índice (Hernández et al., 2018); por su parte 
el diagnóstico de la calidad ecológica de escenarios hídricos afectados por minería a cielo abierto en el corregimiento de Jigualito-Chocó y cuatro fuentes hídricas con distintos tiempos de alteración, evidencia que esta actividad tiene efecto con la concentración de nitrógeno $\left(\mathrm{NO}_{2}\right)$, la temperatura del agua, el caudal y la concentración de oxígeno, estos aspectos y según Vargas-Porras (2016), permiten inferir que la concentración de material suspendido, en combinación con la exposición a la radiación asociada a la deforestación y la cantidad de agua determinan la calidad ambiental de los escenarios hídricos perturbados por minería.

La estimación del índice de calidad ecológica combina el potencial del análisis del gradiente directo junto con un modelo de promedios ponderados, en este marco los puntajes óptimos y de tolerancia brindan una referencia de los valores de bioindicación asociados a la abundancia del grupo biológico de interés (Riss et al., 2002), estos análisis han sido propuestos como métodos efectivos para el desarrollo de índices biológicos (MADS, 2018). Variaciones inesperadas en la composición y estructura de las comunidades de los organismos pueden interpretarse como signos evidentes de algún tipo de alteración (Rossaro et al., 2007). Por esta razón, los cambios significativos en las abundancias de los organismos que presenten altos valores óptimos y altas o bajas tolerancias, así como dominancia de aquellos que obtuvieron mayor tolerancia, indican que el gradiente ambiental presentaría variaciones $\mathrm{y}$, en consecuencia, la forma en la que se distribuyen los organismos es diferente.

Por lo tanto, la detección de los bioindicadores y las variables que caracterizan cada sistema es importante, ya que determinan si los ambientes cambian de estado y requieren medidas de manejo inmediatas o la preservación de las condiciones en el momento (Vasiljević et al., 2017), por ejemplo, los organismos que registran altos óptimos y al mismo tiempo baja tolerancia representan enfoques importantes en el seguimiento del ambiente analizado, en el caso del río San Juan, los organismos como Terspsinoe sp., Borzia sp., Zygnema sp., Chaetophora sp., y Gomphonema sp., corresponden a formas unicelulares y filamentosas y su ecología sugiere amplia distribución en aguas con bajas concentraciones de nutrientes, turbidez y altas concentraciones de oxígeno (Griffith, Hill, Herlihy, \& Kaufmann, 2002; Montoya-Moreno \& Aguirre-Ramírez, 2008; Poulíčková, Hašler, Lysáková, \& Spears, 2008), lo cual concuerda con la indicación final del índice, que sugiere que la baja concentración y el adecuado balance de iones disueltos aumenta la respuesta óptima de los organismos mencionados.

Con relación al grupo de las Quebradas Jigualito los taxa Pinnularia viridis, Actinotanium sp. y Fragillariforma sp., cumplen la misma tendencia de indicación que la descrita en el Río San Juan, estas tres diatomeas corresponden a formas de nivel celular elongado con exoesqueleto de sílice, específicamente presentan formas conspicuas y proyectadas con una importante dimensión lineal axial, que finalmente indican adaptación a baja disponibilidad de luz, dado que proyectan su relación superficie volumen para hacer más eficiente su fisiología (Castro-Roa \& Pinilla-Agudelo, 2014). Por último, en la ciénaga de Ayapel la mayoría de los taxa fitoplanctónicos registran reducidas tolerancias con respecto a su óptimo y entre ellos, las clorofíceas Monorraphidium contortum, Closterium setaceum y la diatomea Nitzschia acicularis se destacan, estos taxa se caracterizan por ser altamente conspicuos y sensibles a la eutroficación (Hansson, 1990; Ramírez, 2000) lo cual concuerda con el comportamiento de las variables que se relacionan con el índice, puesto que se demostró que la alta concentración de nutrientes está asociada con bajos valores del índice de calidad ecológica.

Como conclusiones se considera que el cálculo del ICE permite identificar variables, organismos y ordenamientos numéricos que posibilitan analizar el estado ecológico en un ecosistema, resultados útiles en el diagnóstico y seguimiento de los recursos naturales. Adicionalmente, la obtención de los óptimos y tolerancias permite identificar patrones de desempeño ecológico en cada uno de los sistemas, a partir de ésta información variaciones 
inesperadas en la composición y estructura de las comunidades de los organismos puede interpretarse como signos de algún tipo de alteración (Mora, 2019). Esta investigación motiva a generar plataformas de seguimiento a través de cálculos numéricos que suministren información ecológica para la interpretación y conservación de los ecosistemas acuáticos en Colombia.

Declaración de ética: los autores declaran que todos están de acuerdo con esta publicación y que han hecho aportes que justifican su autoría; que no hay conflicto de interés de ningún tipo; y que han cumplido con todos los requisitos y procedimientos éticos y legales pertinentes. Todas las fuentes de financiamiento se detallan plena y claramente en la sección de agradecimientos. El respectivo documento legal firmado se encuentra en los archivos de la revista.

\section{RESUMEN}

Introducción: La investigación y el diagnóstico de fuentes hídricas es de interés académico y gubernamental, la exploración de instrumentos numéricos aplicados al ordenamiento de cuencas brinda la posibilidad de identificar dónde y qué variables son útiles en programas de monitoreo y rehabilitación en ecosistemas acuáticos, lo cual incluye la calidad del agua, convencionalmente analizada por índices fisicoquímicos e hidrobiológicos. En el 2014 a través de la guía de ordenamiento hídrico en Colombia, se desarrolló una evaluación del índice de calidad ecológica (ICE); la cual genera un sistema numérico de correlaciones que diagnostica, clasifica y detecta afectaciones ambientales. Objetivo: Esta investigación pretende demostrar que el ICE permite evaluar la calidad de ecosistemas acuáticos afectados por diferentes situaciones ambientales. Métodos: Se analizaron tres escenarios ubicados en diferentes regiones de Colombia y como grupo hidrobiológico indicador se utilizaron las algas perifíticas y fitoplanctónicas. Los ecosistemas fueron monitoreados entre el 2007 y 2016 y corresponden a una planicie de inundación, un río andino de alta montaña y un grupo de quebradas en un bosque húmedo tropical. Resultados: Las correlaciones canónicas fueron significativas $(\mathrm{P}<0.005)$ y se estimó el óptimo y la tolerancia de cada taxa; variables relacionadas con la concentración de iones, la amortiguación de la acidez, la temperatura y la hidráulica, incidieron en la distribución de las abundancias de los organismos y la clasificación ecológica mediante el índice. Conclusiones: La aplicación del índice permite identificar variables, organismos y ordenamientos numéricos que posibilitan clasificar el estado ecológico en un sistema, resultados útiles en el diagnóstico y seguimiento de los ecosistemas acuáticos estudiados y que pueden ser implementados con otros escenarios.

Palabras clave: calidad ecológica; algas perifíticas; algas fitoplanctónicas; indicadores ecológicos.

\section{REFERENCIAS}

Barakat, A., El Baghdadi, M., Rais, J., Aghezzaf, B., \& Slassi, M. (2016). Assessment of spatial and seasonal water quality variation of Oum Er Rbia River (Morocco) using multivariate statistical techniques. International Soil and Water Conservation Research, 4(4), 284-292. DOI: 10.1016/j.iswcr.2016.11.002

Braak, C.J.F., \& Prentice, I.C. (1988). A Theory of Gradient Analysis. Advances in Ecological Research, 18, 271-317. DOI: 10.1016/S0065-2504(08)60183-X

Braak, C. J.F., \& Šmilauer, P. (2002). CANOCO Reference Manual and CanoDraw for Windows User's Guide: Software for Canonical Community Ordination (Version 4.5). Microcomputer Power. Ithaca, USA. Retrieved from www.canoco.com

Castro-Roa, D., \& Pinilla-Agudelo, G. (2014). Periphytic diatom index for assessing the ecological quality of the Colombian Andean urban wetlands of Bogotá. Limnetica, 33(2), 297-312.

Chalar, G., Arocena, R., Pacheco, J.P., \& Fabián, D. (2011). Trophic assessment of streams in Uruguay: a trophic State Index for Benthic Invertebrates (TSI-BI). Ecological Indicators, 11(2), 362-369.

Esteves, F. (1998). Fundamentos de limnologia. São Paulo, Brazil: Interciência.

Ettl, H., Gerloff, J., \& Heynig, H. (1977). Süsswasserflora von Mitteleuropa. Jena, Germany: Gustav Fisher.

Flórez-Córdoba, E. (2019). Interpretación Ambiental del Índice de Calidad Ecológica como Insumo para la Gestión Integral del Recurso Hidrico (Tesis de maestría). Universidad de Antioquia, Colombia.

Forero, L.C., Longo, M., Ramírez, R., Jairo, J., \& Chalar, G. (2014). Índice de calidad ecológica con base en macroinvertebrados acuáticos para la cuenca del río Negro (ICE RN-MAE), Colombia. Revista de Biologia Tropical, 62, 233-247.

Global Water Partnership. (2009). Manual para la Gestión Integrada de Recursos Hídricos en Cuencas. Stockholm, Sweden: International Network of Basin Organizations.

Griffith, M.B., Hill, B.H., Herlihy, A.T., \& Kaufmann, P.R. (2002). Multivariate analysis of periphyton assemblages in relation to environmental gradients in Colorado 
Rocky Mountain streams 1. Journal of Phycology, 38(1), 83-95.

Haase, R., \& Nolte, U. (2008). The invertebrate species index (ISI) for streams in southeast Queensland, Australia. Ecological Indicators, 8(5), 599-613.

Hansson, L. (1990). Quantifying the impact of periphytic algae on nutrient availability for phytoplankton. Freshwater Biology, 24(2), 265-273.

Hernández, A.E., Sepúlveda, S.R., Grajales, V.H., Aguirre, R.N., Vélez, M.F., Caicedo, Q.O., Mesa, V.J.A., Cortés, F., \& Montoya, M.Y. (2018). Índice de calidad ecológico empleando algas perifíticas en un tramo del Río San Juan (Antioquia-Colombia). Revista MVZ Córdoba, 23(S), 6998-7012. DOI: 10.21897/ rmvz. 1423

Huber-Pestalozzi, G. (1961). Das Phytoplankton des süsswassers: Chlorophyceae (Grünalgen) (Vol. 5). Stuttgart, Germany: Schweizerbart.

Komárek, J., Anagnostidis, K., Pascher, A., Ettl, H., \& Büdel, B. (1999). Süßwasserflora von Mitteleuropa. Jena, Germany: Gustav Fisher.

MADS. (2014). Guía técnica para la formulación de planes de ordenación y manejo de cuencas hidrográficas. Bogotá, Colombia: Ministerio de Ambiente y Desarrollo Sostenible.

MADS. (2018). Guía para el ordenamiento del recurso hídrico continental superficial. Bogotá, Colombia: Ministerio de Ambiente y Desarrollo Sostenible.

MADVT. (2010). Política nacional para la gestión integral del recurso hídrico. Bogotá, Colombia: Imprenta Nacional de Colombia.

Montoya-Moreno, Y., \& Aguirre-Ramírez, N. (2008). Asociación de algas perifíticas en raíces de macrófitas en una ciénaga tropical Colombiana. Hidrobiológica, 18(3), 189-197.

Mora, F. (2019). The use of ecological integrity indicators within the natural capital index framework: The ecological and economic value of the remnant natural capital of México. Journal for Nature Conservation, 47, 77-92. DOI: 10.1016/j.jnc.2018.11.007
Poulíčková, A., Hašler, P., Lysáková, M., \& Spears, B. (2008). The ecology of freshwater epipelic algae: an update. Phycologia, 47(5), 437-450.

Prescott, G.W., Croasdale, H.T., Vinyard, W.C., \& Bicudo, C.E. de M. (1981). A synopsis of North American desmids. Part II. Desmidiaceae: Placodermae. Lincoln, USA: University Nebraska Press.

Ramírez, J.J. (2000). Fitoplancton de agua dulce: aspectos ecológicos, taxonómicos y sanitarios. Medellín, Colombia: Editorial Universidad de Antioquia.

Riss, W., Ospina, R., \& Gutiérrez, J.D. (2002). Establecimiento de valores de bioindicación para macroinvertebrados acuáticos de la sabana de Bogotá. Caldasia, 42(1), 135-156.

Ros, J. (1979). Prácticas de ecología. Barcelona, España: Ediciones Omega, S.A.

Rossaro, B., Marziali, L., Cardoso, A.C., Solimini, A., Free, G., \& Giacchini, R. (2007). A biotic index using benthic macroinvertebrates for Italian lakes. Ecological Indicators, 7(2), 412-429.

Vargas-Porras, L. (2016). Diagnóstico de la Calidad Ecológica a través de Algas Perifiticas en Escenarios Hídricos Afectados por Minería a Cielo Abierto con Diferente Temporalidad, Jigualito (Condoto, Choco) (Tesis de maestría). Universidad de Antioquia, Colombia.

Vasiljević, B., Simić, S.B., Paunović, M., Zuliani, T., Krizmanić, J., Marković, V., \& Tomović, J. (2017). Contribution to the improvement of diatom-based assessments of the ecological status of large riversThe Sava River Case Study. Science of the Total Environment, 605-606, 874-88. DOI: 10.1016/j. scitotenv.2017.06.206

Waas, T., Hugé, J., Block, T., Wright, T., Benitez-Capistros, F., \& Verbruggen, A. (2014). Sustainability assessment and indicators: Tools in a decision-making strategy for sustainable development. Sustainability, $6(9), 5512-5534$.

Zabala-Agudelo, A.M. (2016). Diagnóstico Ambiental de la Ciénaga de Ayapel a Través de la Morfología Funcional y un Indicador de Calidad Ecológica del Fitoplancton (Tesis de maestría). Universidad de Antioquia, Colombia.

See Digital Appendix at: / Ver Apéndice digital en:

revistas.ucr.ac.cr 